\title{
Haemophilia and acute coronary syndrome - should we still be afraid, should we avoid new antiplatelet drugs?
}

\begin{abstract}
Due to increased life expectancy and unfavorable cardiovascular risk profile in persons with haemophilia (PWH), physicians may face the problem of using potentially contraindicated antiplatelet drugs in this vulnerable to bleedings group of patients. Current European guidelines recommend usage of dual antiplatelet therapy (DAPT) for 12 months after acute coronary syndrome (ACS), whereas haemophilia specialists suggest that such aggressive treatment should be limited to a minimum. The aim of our case report was to assess whether ticagrelor could be a component of DAPT in PWH presenting ACS.

We present the case of 67-years-old male with severe haemophilia A and ST-segment elevation myocardial infarction, who required percutaneous coronary intervention with implantation of 5 drug-eluting stents (DES). Due to high risk of stent thrombosis, after thorough discussion with hematologist, we decided to treat the patient with ticagrelor, acetylsalicylic acid and prophylactic doses of factor VIII. Recommended treatment was well tolerated, without angina and bleeding episodes during follow-up.

In our opinion usage of DES and extended DAPT with ticagrelor may be considered in PWH and ACS with elevated risk of restenosis. Concomitant prophylactic doses of clotting factors should ensure the safety of the treatment used as in the general population.
\end{abstract}

\section{Keywords: haemophilia, acute coronary syndrome, myocardial infarction, ticagrelor, antiplatelet drugs}

\section{Introduction}

Due to improvements in care of persons with haemophilia (PWH), their life expectancy has almost increased to values observed in general population [1]. Although mortality from cardiovascular diseases (CVD) in PWH probably is lower [2,3], they have more unfavorable CVD risk profile than observed in unaffected males [4]. These will certainly lead to the fact that physicians will have to face the problem of using potentially contraindicated antiplatelet drugs in this vulnerable to bleedings patients. According to current European Society of Cardiology (ESC) guidelines for the management of acute coronary syndromes (ACS) and myocardial revascularization, new-generation drug-eluting stents (DES) are indicated for percutaneous coronary intervention (PCI) in ACS patients. A P2Y12 inhibitor (prasugrel, ticagrelor or clopidogrel - only when prasugrel or ticagrelor are not available or contraindicated) is recommended in addition to acetylsalicylic acid (ASA) and maintained for 12 months [5].
Unfortunately, we have neither guidelines nor randomized trials dedicated to antithrombotic treatment of PWH presenting with ACS and the management of this group of patients is therefore based on the experience from the few case reports and/or opinion of the experts.

Staritz et al. tried to solve this problem and gathered a consensus of 15 haemophilia specialists on the applicability of ESC guidelines to PWH. The authors suggested that invasive treatment of ACS should be performed as soon as possible under adequate clotting factor protection. Radial rather than femoral access for coronarography was generally preferred, due to lower bleeding risk. Bare metal stents (BMS) were preferred to DES, principally due to shorter requirement for dual antiplatelet treatment (DAPT) duration which should be limited to a minimum. Trough levels of clotting factors should be around $50 \%$ within $24 \mathrm{~h}$ of PCI and minimum trough levels should not fall below $5-15 \%$ on DAPT [6].
Michal Kacprzak ${ }^{* 1}$, Magdalena Brzeczek ${ }^{1}$, Wlodzimierz Koniarek ${ }^{1}$, Marzenna Zielinska ${ }^{1}$ \& Krzysztof Chojnowski ${ }^{2}$

${ }^{1}$ Intensive Cardiac Therapy Clinic, Medical University of Lodz, Poland

${ }^{2}$ Department of Hemostasis, Medical University of Lodz, Poland

*Author for correspondence:

michal.kacprzak@umed.lodz.pl 
Clinical data regarding the use of new antiplatelet drugs (ticagrelor and prasugrel) in PWH are even more limited. Thus, the aim of our case report was to assess whether ticagrelor could be a component of DAPT in PWH presenting with ACS.

\section{Case report}

A 67-years-old male with severe haemophilia A, treated with concentrated factor VIII (FVIII) only during bleeding (last dose given 8 days before), chronic hepatitis $\mathrm{C}$, suffering from recurrent, retrosternal pain at rest since 5 days, was admitted to our clinic with the suspicion of ST-segment elevation myocardial infarction (STEMI). The patient negated arterial hypertension, diabetes mellitus, cigarette smoking and previous history of angina.

An ECG revealed sinus rhythm, pathological Q-waves in V1-V3 leads, persistent ST-segment elevation in V3-V4 leads and negative T-waves in I, aVL, V2-V5 leads. After urgent consultation with hematologist, the patient received 4000 IU of FVIII iv (Beriate, CSL Behring GmbH, Germany), 300mg of ASA, $600 \mathrm{mg}$ of clopidogrel and $5000 \mathrm{IU}$ of unfractionated heparin iv. Rosuvastatin and continuous IV flow of nitroglycerin were also given. Urgent coronary angiography, using the right radial access, revealed advanced, critical stenoses of the left anterior descending artery and diagonal branch (FIGURE 1). Due to multi-level lesions in relatively narrow coronary arteries, the operator decided to implant 5 DES: 4 stents eluting everolimus and 1 eluting biolimus A9, obtaining optimal widening of the vessels, without residual stenosis and dissection. Echocardiography showed hypokinesis of anterior wall, apex and interventricular septum, with mild systolic left ventricular dysfunction (ejection fraction $=46 \%$ ). After 12 hours from the first dose the patient received another 3000 IU of FVIII. Because of high risk of stent thrombosis, we decided to use ticagrelor (loading dose $180 \mathrm{mg}$ ) instead of clopidogrel.

During the following days of hospitalization, the patient received daily 1000 to $1500 \mathrm{IU}$ of FVIII, maintaining acceptable FVIII activity level (peak level: $176 \%$ after the first dose, trough level: $45 \%$ on the third day). We did not observe any bleeding complications. On the fifth day the patient was discharged on ASA (75mg QD), ticagrelor (90 mg BID, with the

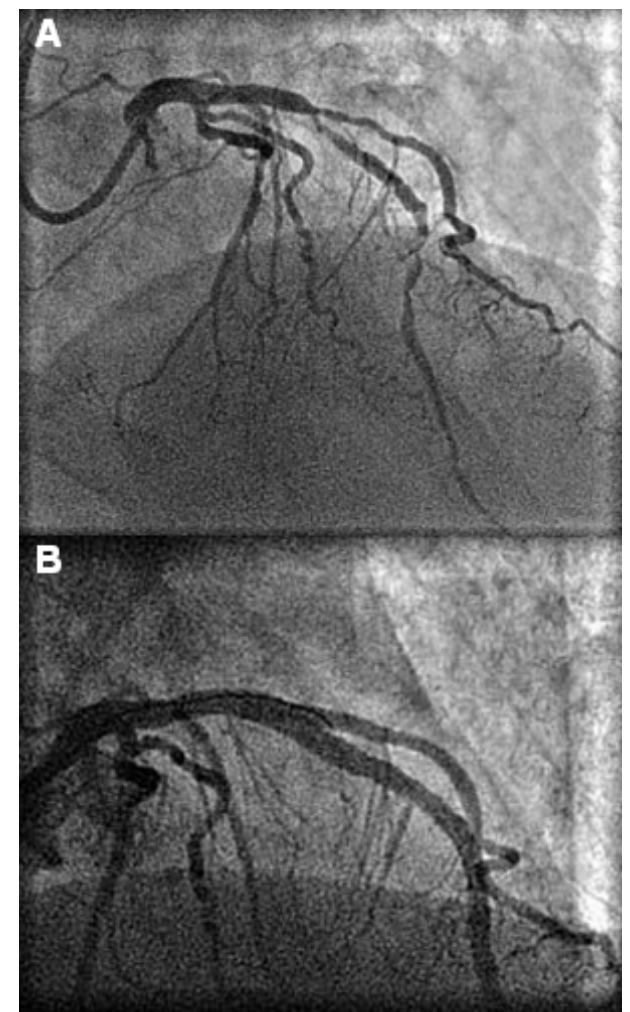

FIGURE 1. Coronary angiography of left coronary artery: (A) before and (B) after $\mathrm{PCI}$.

intention of use by 12 months), pantoprazole (40mg QD), rosuvastatin, bisoprolol, ramipril. After thorough discussion with hematologist, to ensure the safety of treatment with DAPT, we decided to give the patient 2000 IU QD of FVIII for the time of DAPT, with FVIII activity level $\geq 30 \%$. After 12 months we decided to withdraw ticagrelor, leaving the patient on ASA treatment, with 2500 IU of FVIII administered every two days, which allowed maintaining FVIII activity $\geq 5 \%$.

After 30 months of follow-up recommended treatment was well tolerated, without angina and bleeding episodes.

\section{Discussion}

To our best knowledge, this is the first described patient with severe haemophilia A and STEMI treated with PCI with DES implantation and subsequent extended ticagrelor treatment.

Although ticagrelor and prasugrel are usually contraindicated in $\mathrm{PWH}$, treatment with ticagrelor in patients with ACS significantly reduces the rate of death from vascular causes, myocardial infarction or stroke, without a significant risk in major bleeding compared to clopidogrel [7]. In our patient - due to long 
sections $(92 \mathrm{~mm})$ of relatively narrow coronary arteries coated with DES and high risk of stent thrombosis, we decided to use ticagrelor as a component of DAPT.

Fefer et al. presented 3 cases of PWH with ACS treated invasively and review of the available literature of 17 similar cases, but none of the patients received ticagrelor. Although the usage of antithrombotic drugs and the duration of antiplatelet therapy varied widely between patients (none vs. 9 months of DAPT), reported outcomes were generally favorable [8].

Fogarty et al. in their 10 -year, retrospective survey, comprising 2380 adult PWH, stated only 20 episodes of ACS in 19 patients. As in the previous paper, none of the patients received new antiplatelet drugs. Among 7 patients receiving ASA and clopidogrel, the duration of DAPT ranged from $<1$ to 12 months. Also FVIII trough levels during secondary prophylaxis varied from $3-5 \%$ to $\geq 30 \%$. Hemorrhagic complications were observed in 2 of 17 patients receiving antiplatelet drugs [9].

Three patients treated with ticagrelor during PCI, switched to clopidogrel after coronary intervention were described by Tuinenburg et al. But patients from their group had mild or moderate haemophilia and BMS were used during coronary intervention [10].
A case of patient with acquired mild haemophilia presenting with STEMI treated with PCI was described by Routledge et al. The patient was treated for two months with ticagrelor and ASA. Due to relatively high levels of FVIII ( 25 to >40 IUdL-1), she did not receive any clotting factor concentrates [11].

Reddy et al. presented a case of patient with mild haemophilia A and STEMI treated with PCI with BMS. The patient was loaded with prasugrel, but discharged on ASA and clopidogrel for 1 month with prophylactic tranexamic acid during DAPT [12].

\section{Conclusion}

Taking into account very few available case reports and uncomplicated course of treatment of our patient, in our opinion usage of newgeneration DES and extended DAPT with ticagrelor may be considered in PWH and ACS with elevated risk of restenosis. Concomitant prophylactic doses of FVIII should ensure the safety of the treatment used as in the general population. However, this approach requires not only close cooperation between cardiologists and hematologists, but also improvement in access to FVIII therapy - from on-demand to preventive treatment of PWH after ACS treated invasively. 


\section{REFERENCES}

Mejia-Carvajal C, Czapek EE, Valentino LA. Life expectancy in hemophilia outcome. J. Thromb. Haemost. 4, 507-509 (2006).

Darby SC, Kan SW, Spooner RJ, et al. Mortality rates, life expectancy, and causes of death in people with hemophilia A or $B$ in the United Kingdom who were not infected with HIV. Blood. 110, 815-825 (2007).

Biere-Rafi S, Tuinenburg A, Haak BW, et al. Factor VIII deficiency does not protect against atherosclerosis. J. Thromb. Haemost. 10, 30-37 (2012).

Fransen van de PDE, Fischer K, Makris $M$, et al. Unfavourable cardiovascular disease risk profiles in a cohort of Dutch and British haemophilia patients. J. Thromb. Haemost. 109, 16-23 (2013).

Windecker S, Kolh P, Alfonso F, et al. 2014 ESC/EACTS Guidelines on myocardial revascularization: The Task Force on Myocardial Revascularization of the European Society of Cardiology (ESC) and the European Association for CardioThoracic Surgery (EACTS) Developed with the special contribution of the European Association of Percutaneous Cardiovascular Interventions (EAPCI). Eur. Heart J. 35, 2541-2619 (2014).

Staritz P, de Moerloose P, Schutgens R, et al. Applicability of the European Society of Cardiology guidelines on management of acute coronary syndromes to people with haemophilia - an assessment by the ADVANCE Working Group. Haemophilia. 19, 833-840 (2013).

Wallentin L, Becker RC, Budaj A, et al. Ticagrelor versus clopidogrel in patients with acute coronary syndromes. N. Engl. J. Med. 361, 1045-1057 (2009).

Fefer P, Gannot S, Lubetsky A, et al. Percutaneous coronary intervention in patients with haemophilia presenting with acute coronary syndrome: an interventional dilemma: case series, review of the literature, and tips for management. J. Thromb. Thrombolysis. 35, 271-278 (2013).
Fogarty PF, Mancuso ME, Kasthuri R, et al. Global Emerging Hemophilia Panel (GEHEP). Presentation and management of acute coronary syndromes among adult persons with haemophilia: results of an international, retrospective, 10-year survey. Haemophilia. 21, 589-597 (2015).

Tuinenburg A, Damen SA, Ypma PF, et al. Cardiac catheterization and intervention in haemophilia patients: prospective evaluation of the 2009 institutional guideline. Haemophilia. 19, 370-377 (2013).

Routledge DJ, Fraser D, Thachil J, et al. Management of a myocardial infarction in a patient with classical acquired haemophilia patient in partial remission. Haemophilia. 21, e494-e496 (2015).

Reddy SA, Hoole SP, Besser MW. Primary percutaneous coronary intervention in a patient with haemophilia A. Case Rep. Med. 189796 (2013).

This special issue on Current Trends in Clinical Research was edited by Dr. Mohamed Elsayed. 